NOTICE: this is the author's version of a work that was accepted for publication in International Journal of Fatigue. Changes resulting from the publishing process, such as peer review, editing, corrections, structural formatting, and other quality control mechanisms may not be reflected in this document. Changes may have been made to this work since it was submitted for publication. A definitive version was subsequently published in International Journal of Fatigue, [VOL 73, (April 2015)] DOI 10.1016/j.ijfatigue.2014.11.013

\title{
Analysis of Ultra Low Cycle Fatigue problems with the Barcelona plastic damage model and a new isotropic hardening law
}

\author{
X. Martinez ${ }^{a, b},{ }^{*}$, S. Oller ${ }^{a, c}$, L.G. Barbu ${ }^{a, c}$, A.H. Barbat ${ }^{a, c}$ and A.M.P. de Jesus ${ }^{d, e}$ \\ a. International Center for Numerical Methods in Engineering (CIMNE). Campus Nord, Gran Capità s/n, 08034 Barcelona, Spain \\ b. Department of Nautical Science and Engineering - UPC. Pla de Palau 18, 08003 Barcelona, Spain \\ c. Department of Strenght of Materials and Structural Engineering - UPC. Edifici C1, Campus Nord, Jordi Girona 1-3, 08034 \\ Barcelona, Spain \\ d. IDMEC, Rua Dr. Roberto Frias, 4200-465 Porto, Portugal \\ e. Departamento de Engenharia Mecânica, Faculdade de Engenharia, Universidade do Porto, Rua Dr. Roberto Frias, 4200-465 \\ Porto, Portugal \\ *Corresponding author. x.martinez@upc.edu
}

Key words: Ultra low cycle fatigue; plastic damage; isotropic hardening; kinematic hardening; constitutive modelling

\section{Abstract}

This paper presents a plastic-damage formulation and a new isotropic hardening law, based on the Barcelona plastic damage model initially proposed by Lubliner et al. in 1989 [1], which is capable of predicting steel failure due to Ultra Low Cycle Fatigue (ULCF). This failure mechanism is obtained when the material is subjected to cyclic loads and breaks after applying a very low number of cycles, usually less than hundreds. The failure is driven by the plastic response of the material, and it is often predicted based on the plastic strains applied to it. The model proposed in this work has been formulated with the objective of predicting accurately the plastic behavior of the material, as well as its failure due to ULCF. This is achieved taking into account the fracture energy dissipated during the whole loading process. This approach allows the simulation of ULCF when it takes place due to regular cyclic loads or non-regular cyclic loads, as it is the case of seismic loads. Several simulations are conducted in order to show the capabilities of the formulation to reproduce the mechanical response of steel when it is subjected to regular and non-regular cyclic loads. The formulation is validated comparing the numerical results with several experimental tests made on X52 steel specimens. The agreement between the numerical and experimental results asses the validity of the proposed model to predict the plastic behavior of steel and its failure due to Ultra Low Cycle Fatigue.

\section{INTRODUCTION}

The mechanical phenomenon known as fatigue consists in the loss of material strength, and consequent failure, due to the effect of cyclic loads. Fatigue is characterized, among other parameters, by the number of cycles, load amplitude and reversion index ([2], [3] and [4]). Material failure is 
produced by an inelastic behaviour, micro-cracking and crack coalescence, which lead to the final collapse of structural parts.

Fatigue phenomenon is defined more generally in the ASTM E1823 standard as: "the process of permanent, progressive and localized structural change which occurs to a material point subjected to strains and stresses of variable amplitudes which produce cracks which lead to total failure after a certain number of cycles" [5]. In this definition it is possible to include all fatigue ranges, from "Ultra Low Cycle Fatigue" (ULCF), to "Low Cycle Fatigue" (LCF) and "High Cycle Fatigue" (HCF).

While there is a general agreement that for failures in the range of $10^{6}$ to $10^{8}$ cycles the structure has failed in the high cycle fatigue range, there is not such agreement in defining the limits for low cycle and ultra low cycle fatigue. Authors such as Kanvinde and Deierlein [6] consider that LCF is found between 100s and 1000s cycles and that ULCF is in the range of 10-20 cycles; and other authors, such as Xue [7], put these limits in $10^{4}$ for LCF and 100 for ULCF. However, despite these discrepancies, there is a general agreement that plastic behaviour of the material plays an important role in the failure due to LCF or ULCF [8].

According to the literature review made by Yao and Munse in [9], first attempts to characterize LCF and ULCF can be attributed to Kommers who, in 1912, conducted several tests on a cantilever specimen subjected to cyclic bending. After these tests he reached the conclusion that the magnitude of deflection plays an important role in low cycle fatigue. However, main efforts to characterize the parameters driving LCF and ULCF are not found until 1950s, when numerous experimental programs where carried out to calibrate the material constants for various metals. A large amount of work is documented from this period. The experimental data is usually plotted on a log-log scale with the abscissa representing the number of life cycles and the ordinate the plastic strain amplitude. This graph is known as the $\Delta \varepsilon^{p}-N$ curve. Following this approach, probably the most know, and most widely used, procedure to predict material failure under LCF and ULCF is the Manson-Coffin law ([8], [10], [11]):

$$
\Delta \varepsilon_{p} \cdot N^{\alpha}=C
$$

$\Delta \varepsilon_{p}$ being the plastic strain range in the material, $N$ the number of cycles that can be applied before ULCF and LCF failure, and $\alpha$ and $C$ material constants.

From this first equation proposed by Coffin and Manson, several authors have provided their own law in order to improve the accuracy on the predicted cycles before failure, especially in the Ultra Low Cycle Fatigue regime. For instance, Xue [7] observed, from experimental results, that the law did not fit well in the range of very low life cycles, less than 100, so he proposed a new exponential damage rule that improved this accuracy. Kuroda [11] also provided a modification on the original Coffin-Manson law in order to predict the failure below 100 cycles. In this case the model is based on the accumulation of damage due to three different effects: tensile straining, cyclic straining and crack propagation. 
The approach used by Tateishi et al. [12] to simulate LCF failure is also interesting. These authors use Miner's rule to couple the effect of high cycle fatigue with the effect of low cycle fatigue, by adding a ductile damage term. This last term depends on the yield strain of the material, the rupture strain and the strain that is applied in a given cycle.

One of the main drawbacks of most of the existing formulations to characterize ULCF and LCF is that they require regular cycles to predict material failure, or they couple the effects of non-regular cycles using the Miner's rule, which requires knowing the performance of the structure under regular cycles. However, this regularity often does not exist. An example of an ULCF failure due to an irregular cyclic load is found in the failure of structures subjected to seismic loads, where the frequency varies along time and each cycle may have different amplitudes.

An interesting approach to characterize low cycle fatigue accounting for non-regular cycles is the one proposed by Jiang et al. [13], which defines an independent continuous cumulative damage function (EVICD) based on the accumulation of plastic strain energy. This formulation is based on previous models of EVICD ([14], [15] and [16]) and states that the total damage can be computed as:

$$
D=\int d D \quad \text { with } \quad d D=\zeta \cdot d W^{p}
$$

being $D$ the fatigue damage, $W^{p}$ the plastic strain energy density and $\zeta$ a function determined experimentally based on the fatigue response of the material. With this approach, the authors obtain an evolution of the fatigue damage parameter as the simulation evolves, the material failure is obtained when $D=1$. In [13], the model is tested for fatigue ranges between $10^{3}$ to $10^{7}$ cycles, which corresponds to low and high cycle fatigue.

Another interesting approach based on damage accumulation is the one proposed by Kanvinde and Deierlein ([6], [17], and [18]). These authors, in order to account for the effects of void growth and coalescence that drive the fracture of metallic materials, propose a model that calculates the void growth and compares it with a critical value to detect material failure. This parameter is obtained experimentally. The initial formulation developed for monotonic cases (Void Growth Model - VGM [17]) is extended to cyclic loads by differentiating the void growth obtained in the tensile and compressive regions of the load cycle. Therefore, the void growth in the Cyclic Void Growth Model (CVGM) can be obtained as [18]:

$$
\begin{aligned}
V G I_{\text {cyclic }}= & \sum_{\text {tensilecycles }} C_{1} \cdot \int_{\varepsilon_{1}}^{\varepsilon_{2}} \exp \left(\left|1.5 \frac{\sigma_{m}}{\sigma_{e}}\right|\right) d \varepsilon_{p}- \\
& -\sum_{\text {comp ressite cycles }} C_{2} \cdot \int_{\varepsilon_{1}}^{\varepsilon_{2}} \exp \left(\left|1.5 \frac{\sigma_{m}}{\sigma_{e}}\right|\right) d \varepsilon_{p}<V G I_{\text {cyclic }}^{\text {critical }}
\end{aligned}
$$

This formulation, as well as the formulation proposed by Jiang et al. [13], are capable to account for regular and non-regular cycles, as both formulations are based on the addition of certain quantities 
while the material increases its plastic strain. However, they both have the drawback of being based on a failure criterion that is completely independent of the plastic model (uncoupled approaches): it is calculated as the simulation advances and, when it reaches a certain level, the criterion tells the code that the material has failed.

The simulation of LCF and ULCF has also been approached using non-linear constitutive laws. This is the case of Saanouni and Abdul-Latif ([19], [20]), who propose the use of a representative volume element (RVE), and a non-linear law based on the slip theory, to account for the dislocation movement of metallic grains. Instead of a RVE, Naderi et al. [21] proposed simulating the progressive failure of a given structural element by applying random properties to the different finite elements in which it is discretized. The constitutive model used to characterize LCF failure is the one defined by Lemaitre and Chaboche in [22]. The use of a stochastic approach is also the approach used by Warhadpande et al. [23], who applied random properties to a Voronoi cell. In most of these models the damage variable is also calculated independently of the non-linear constitutive law used to simulate the material performance.

Current work proposes the use of a plastic damage model, and presents a new isotropic hardening law, to simulate Ultra Low Cycle Fatigue. The model developed is based on the Barcelona model originally formulated by Lubliner et al. ([1], [24], [25] and [26]). Although this model was originally defined to simulate brittle materials such as concrete, here is used with a kinematic and isotropic hardening law specifically defined for the simulation of steel. The isotropic hardening law is defined with an initial hardening region followed by a softening region. One of the main characteristics of the model is that the isotropic hardening behaviour of the material is driven by the plastic energy dissipated: the model measures the fracture energy that is dissipated as the plastic strain increases, and this energy is used to define the plastic strain level at which material softening due to damage starts and finishes. The model considers that damage initiates when the plastic law reaches the softening region and the complete failure is obtained when all fracture energy of the material is dissipated. A first preliminary description of the procedure used by the proposed model has been already presented in [27] and [28].

This work proves that the proposed model it capable of simulating material failure due to Ultra Low Cycle Fatigue by its own, without the need of any other damage variable computed independently of the plastic formulation. Besides, the proposed approach is not only capable of predicting material failure for regular and non-regular cyclic loads, but it is also capable of coupling cyclic loads with monotonic loads, which allows to predict that the structure will fail sooner if the monotonic load is applied after several hysteresis cycles, than if these cycles are not applied. This capability is obtained as a consequence of the fact that the material failure is predicted by the plastic non-linear constitutive equation itself. Another advantage of the formulation proposed is that it is capable of using any yield and potential surfaces to characterize the material, which increases its applicability to different steel alloys. 
The inelastic theory of plasticity can simulate the material behavior beyond the elastic range, taking into account the change in the strength of the material through the movement of the yield surface, representing isotropic and kinematic hardenings. It is assumed that each point of the solid follows a thermo-elasto-plastic constitutive law (stiffness hardening/softening) ([1], [29] and [30]) with the stress evolution depending on the free strain variable and plastic internal variables. The formulation presented hereafter studies the phenomenon of stiffness degradation accumulation through a plasticdamage law.

\subsection{Plastic Model}

Since this work is guided to mechanical problems with small elastic strains and large inelastic strains, the free energy additively hypothesis is accepted $\psi=\psi^{e}+\psi^{p}$ ([31], [32]). The elastic $\psi^{e}$ and plastic $\psi^{p}$ parts of the free energy are written, in the reference configuration for a given entropy $\eta$ and temperature $\theta$ field, as the elastic Green strains $E_{i j}^{e}=E_{i j}-E_{i j}^{p}-E_{i j}^{\theta}$; the two last variables operate as free field variables ([29], [32] and [33]). The free energy is thus written as,

$$
\psi=\psi^{e}\left(E_{i j}^{e}, \theta\right)+\psi^{p}\left(\gamma^{p}, \theta\right)=\left\{\frac{1}{2 m}\left[E_{i j}^{e} \cdot C_{i j k l} \cdot E_{k l}^{e}\right]+\psi^{p}\left(\gamma^{p}, \theta\right)\right\}-\theta \cdot \eta
$$

Considering the second thermodynamics law (Clausius-Duhem inequality, [31], [34] and [35]), the thermo mechanical dissipation can be obtained as [32]:

$$
\Xi=\frac{S_{i j} \dot{E}_{i j}^{p}}{m}-\frac{\partial \psi}{\partial \gamma^{p}} \dot{\gamma}^{p}-\frac{1}{\theta m} q_{i} \nabla \theta \geq 0
$$

The accomplishment of this dissipation condition, equation (5), demands that the expression of the stress and the entropy should be defined as (Coleman method; see [35]);

$$
S_{i j}=m \frac{\partial \psi^{e}}{\partial E_{i j}^{e}}=C_{i j k l} \cdot E_{k l}^{e} ; \quad \eta=-\frac{\partial \psi}{\partial \theta}
$$

From the last expression is possible to obtain the general expression of the tangent constitutive tensor,

$$
C_{i j k l}^{t}=\frac{\partial S_{i j}}{\partial E_{i j}^{e}}=m \frac{\partial^{2} \psi^{e}}{\partial E_{i j}^{e} \partial E_{k l}^{e}}
$$

In equations (4) to (7), $m$ is the material density, $E_{i j}, E_{i j}^{e}, E_{i j}^{p}$ are the total, elastic and plastic strain tensors, respectively, $S_{i j}$ is the stress tensor for a single material point, $C_{i j k l}$ and $C_{i j k l}^{t}$ are the initial and tangent constitutive tensors, and $\gamma^{p}$ are the plastic internal variables. 


\subsection{Yield plastic functions}

The yield function $F$ accounts for the residual strength of the material, which depends on the current stress state, the temperature and the plastic internal variables. This $F$ function and the plastic potential $G$ have the following form, taking into account isotropic and kinematic plastic hardening (Bauschinger effect; [22], [36] and [37]),

$$
\begin{aligned}
& F\left(S_{i j}, \alpha^{p}, \theta\right)=f\left(S_{i j}-\alpha_{i j}\right)-K\left(S_{i j}, \kappa^{p}, \theta\right) \leq 0 \\
& G\left(S_{i j}, \alpha^{p}, \theta\right)=g\left(S_{i j}-\alpha_{i j}\right)=c t e
\end{aligned}
$$

where $f\left(S_{i j}-\alpha_{i j}\right)$ and $g\left(S_{i j}-\alpha_{i j}\right)$ are the uniaxial equivalent stress functions depending on the current value of the stresses, $S_{i j}$, and the kinematic plastic hardening internal variable, $\alpha_{i j}$; $K\left(S_{i j}, \kappa^{p}, \theta\right)$ is the plastic strength threshold, $\kappa^{p}$ is the plastic isotropic hardening internal variable, and $\theta$ is the temperature at current time $t$ ([1], [29] and [30]).

The evolution law for the plastic strain is obtained from the evolution of the plastic potential as,

$$
\dot{E}_{i j}^{p}=\dot{\lambda} \frac{\partial G^{p}}{\partial S_{i j}}
$$

where $\dot{\lambda}$ is the plastic consistency parameter. We will talk of associated plasticity when the plastic potential is the same as the plastic yield function, which is the assumption followed in this research.

\subsection{Kinematic Hardening}

Kinematic hardening accounts for a translation of the yield function allowing the representation of the Bauschinger effect in the case of cyclic loading. A two dimensional representation of this movement in the $S_{1}-S_{2}$ plane is shown in the following figure:

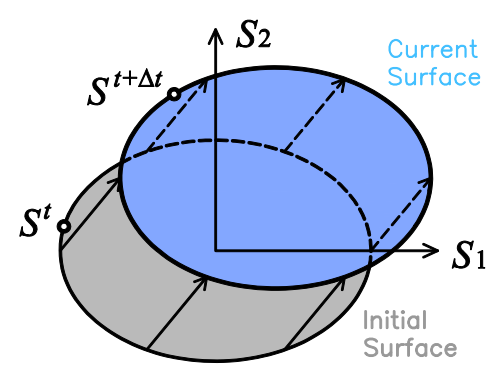

Figure 1. Translation of the yield surface as a result of kinematic hardening

This translation is driven by the kinematic hardening internal variable $\alpha_{i j}$ which, in a general case, varies proportionally to the plastic strain of the material point $([22],[38])$. There are several laws that 
define the evolution of this parameter. Current work uses a non-linear kinematic hardening law, which can be written as:

$$
\dot{\alpha}_{i j}=c_{k} \dot{E}_{i j}^{P}-d_{k} \alpha_{i j} \dot{p}
$$

where $c_{k}$ and $d_{k}$ are material constants, $E_{i j}^{p}$ is the plastic strain, and $\dot{p}$ is the increment of accumulated plastic strain, which can be computed as: $\dot{p}=\sqrt{2 / 3 \cdot \dot{E}_{i j}^{p}: \dot{E}_{i j}^{p}}$. Note that the $2 / 3$ is valid in case of using Von-Mises as the actual yield surface. In other cases, this value should be modified.

\subsection{Isotropic Hardening}

Isotropic hardening provides an expansion or a contraction of the yield surface. The expansion corresponds to a hardening behaviour and the contraction to a softening behavior. In the following figure a two dimensional representation of this effect in the $S_{1}-S_{2}$ plane is depicted:

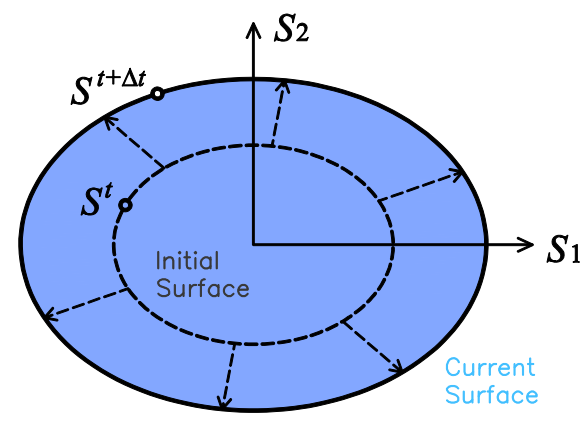

Figure 2. Expansion of the yield surface as a result of isotropic hardening

The evolution of isotropic hardening is controlled by the evolution of the plastic hardening function $K$, which is often defined by an internal variable $\kappa^{p}$. The rate equation for these two functions may be defined, respectively:

$$
\begin{aligned}
& \dot{K}=\dot{\lambda} \cdot H_{k}=h_{k} \cdot \dot{\kappa}^{p} \\
& \dot{\kappa}^{p}=\dot{\lambda} \cdot H_{\mathbf{k}}=\dot{\lambda} \cdot\left[h_{\mathrm{k}}: \frac{\partial G}{\partial S}\right]=h_{\mathrm{k}} \cdot \dot{E}^{p}
\end{aligned}
$$

where $k$ denotes scalar and $\mathbf{k}$ states for a tensor function. Depending on the functions defined to characterize these two parameters different material performances can be obtained. A new function to characterize metallic materials is proposed in this work and described in Section 3 of this document. 


\subsection{Stress-strain relation and consistency factor}

Once the material has exceeded its yield threshold stress, the stress-strain relation is defined by the tangent stiffness tensor. The expression of this tensor, as well as the expression of the plastic consistency parameter can be obtained from the plastic yield criterion and the Prager consistency condition [38]:

$$
\left.\begin{array}{l}
F\left(S_{i j}, \alpha^{p}, \theta\right)=f\left(S_{i j}-\alpha_{i j}\right)-K\left(S_{i j}, \kappa^{p}, \theta\right)=0 \\
\dot{F}=\frac{\partial F}{\partial S} \dot{S}+\frac{\partial F}{\partial \alpha} \dot{\alpha}+\frac{\partial F}{\partial K} \dot{K}=0
\end{array}\right\} \frac{\partial F}{\partial S} \dot{S}+\frac{\partial F}{\partial \alpha} \dot{\alpha}-\dot{K}=0
$$

Using previous expressions, it is possible to rewrite equation (12) as:

$$
\frac{\partial F}{\partial S}: C:\left(\dot{E}-\dot{E}^{p}\right)+\frac{\partial F}{\partial \alpha}:\left(c_{k} \dot{E}_{i j}^{P}-d_{k} \alpha_{i j} \dot{p}\right)-h_{k}\left(h_{\mathbf{k}}: \dot{E}^{p}\right)=0
$$

From this expression it is possible to obtain the consistency factor using equation (9):

$$
\frac{\partial F}{\partial S}: C: \dot{E}-\frac{\partial F}{\partial \alpha}:\left(d_{k} \alpha_{i j} \dot{p}\right)-\dot{\lambda} \cdot\left[\frac{\partial F}{\partial S}: C: \frac{\partial G}{\partial S}-c_{k} \frac{\partial F}{\partial \alpha}: \frac{\partial G}{\partial S}+h_{k} \cdot h_{\mathbf{k}}: \frac{\partial G}{\partial S}\right]=0
$$

Therefore:

$$
\dot{\lambda}=\frac{\frac{\partial F}{\partial S}: C: \dot{E}-\frac{\partial F}{\partial \alpha}:\left(d_{k} \alpha_{i j} \dot{p}\right)}{\frac{\partial F}{\partial S}: C: \frac{\partial G}{\partial S}-c_{k} \frac{\partial F}{\partial \alpha}: \frac{\partial G}{\partial S}+h_{k} \cdot h_{\mathbf{k}}: \frac{\partial G}{\partial S}}
$$

The tangent stiffness tensor relates the total strain rate to the stress rate:

$$
\dot{S}=C^{t}: \dot{E}
$$

Finally, the expression of the tangent stiffness tensor can be obtained from the consistency factor:

$$
C^{t}=C-\frac{\left[C: \frac{\partial G}{\partial S}\right] \otimes\left[\frac{\partial F}{\partial S}: C\right]}{\frac{\partial F}{\partial S}: C: \frac{\partial G}{\partial S}-c_{k} \frac{\partial F}{\partial \alpha}: \frac{\partial G}{\partial S}+h_{k} \cdot h_{\mathbf{k}}: \frac{\partial G}{\partial S}}
$$

It has to be noted that expression (17) has been obtained disregarding the non-linear term of kinematic hardening. Despite having a first approximation of the analytical expression that provides the tangent stiffness tensor, in many occasions the calculation of the partial derivatives of the yield and potential functions is not straightforward. In those cases, a numerical derivation can be performed. This procedure, although expensive, provide an accurate approximation that improves the global convergence of the problem. An efficient procedure to conduct this numerical derivation, as well as the 
advantages obtained with it, are shown in [39]. A detailed description of the integration procedure of the constitutive equations can be found in references [32] and [38].

\section{NEW ISOTROPIC HARDENING LAW}

Equation (11) allows the incorporation of different hardening laws to describe the material performance. In the Barcelona model defined in [1], the laws defined are driven by the fracture energy of the material. This work presents a new law, specially developed for steel materials, that has been designed to reproduce their hardening and softening performance under monotonic and cyclic loading conditions. This law also depends on the fracture energy of the material.

\subsection{Fracture Energy}

Classical fracture mechanics defines the fracture energy of a material as the energy that has to be dissipated to open a fracture in a unitary area of the material. This energy is defined as:

$$
G_{f}=\frac{W_{f}}{A_{f}}
$$

where $W_{f}$ is the energy dissipated by the fracture at the end of the process, and $A_{f}$ is the area of the surface fractured. The total fracture energy dissipated, $W_{f}$, in the fracture process can be used to define a fracture energy by unit volume, $g_{f}$, required in a continuum mechanics formulation:

$$
W_{f}=G_{f} \cdot A_{f} \equiv \int_{V_{f}} g_{f} d V
$$

This last equation allows establishing the relation between the fracture energy defined as a material property, $G_{f}$, and the maximum energy per unit volume:

$$
g_{f}=\frac{W_{f}}{V_{f}}=\frac{W_{f}}{A_{f} \cdot l_{f}}=\frac{G_{f}}{l_{f}}
$$

Thus, the fracture energy per unit volume is obtained as the fracture energy of the material divided by the fracture length. This fracture length corresponds to the distance, perpendicular to the fracture area, in which this fracture propagates.

In a real section, this length tends to be infinitesimal. However, in a finite element simulation, in which continuum mechanics is applied to a discrete medium, this length corresponds to the smallest value in which the structure is discretized: the length represented by a gauss point. 
Therefore, in order to have a finite element formulation consistent and mesh independent, it is necessary to define the hardening law in function of the fracture energy per unit volume ([1], [30], [40] and [41]). This value is obtained from the fracture energy of the material, $G_{f}$, and the size of the finite element in which the structure is discretized.

\subsection{Hardening Function and Hardening Internal Variable}

The hardening function defines the stress of the material when it is in the non-linear range. There are many possible definitions that can be used for this function fulfilling equation (11). Among them, here it is proposed to use a function that describes the evolution of an equivalent uniaxial stress state, like the one shown in Figure 3.

This equivalent stress state shown in Figure 3 has been defined to match the uniaxial stress evolution described by most metallic materials. After reaching the yield stress, the curve is divided in two different regions. The first region is defined by curve fitting from a given set of equivalent stressequivalent strain points. The curve used to fit the points is a polynomial of any given order defined using the least squares method. The data given to define this region is expected to provide an increasing function, in order to obtain a good performance of the formulation when performing cyclic analysis.

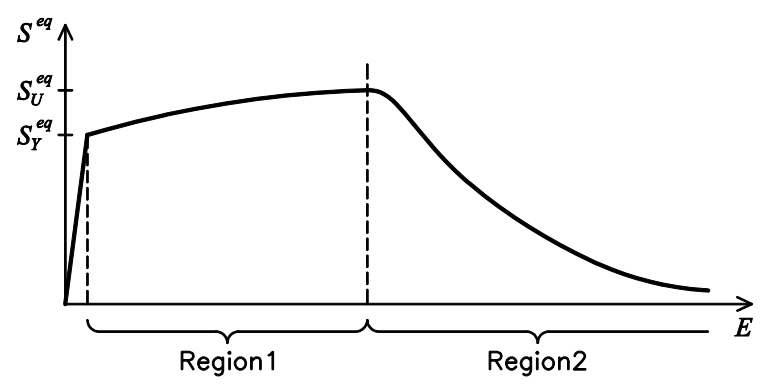

Figure 3. Evolution of the equivalent stress

The second region is defined with an exponential function to simulate softening. The function starts with a null slope that becomes negative as the equivalent plastic strain increases. The exact geometry of this last region depends on the fracture energy of the material. The adjustment of this exponential softening to experimental results is usually very difficult, as the stress drop is very fast and experimental tests cannot capture it. Therefore, its definition will be done just to obtain a more or less steep slope. The selection of an exponential function instead of a straight line or a polynomial is made because the exponential function provides a response that facilitates the numerical convergence.

It has to be noted that the initial plateau that is usually found in monotonic stress-strain graphs of carbon steels is not represented in the stress evolution proposed in this model and, therefore, it is not shown in Figure 3. This is because the definition of this region will lead to inaccurate results when performing cyclic simulations of the material. 
The hardening internal variable, $\kappa^{p}$, accounts for the evolution of the plastic hardening function, $K$. In current formulation $\kappa^{p}$ is defined as a normalized scalar parameter that takes into account the amount of volumetric fracture energy dissipated by the material in the actual strain-stress state. This is:

$$
\kappa^{p}=\frac{1}{g_{f}} \int_{t=0}^{t} S: \dot{E}^{p} d t
$$

Figure 4 shows shaded in green the volumetric fracture energy required by a uniaxial loaded material, for a given plastic strain, $E^{p}$, to start the material softening. The hardening internal variable defined in (21) is calculated normalizing this fracture energy by the total fracture energy of the system, $g_{f}$, which corresponds to the total area below the curve $S^{e q}\left(E^{p}\right)$, shaded with parallel lines.

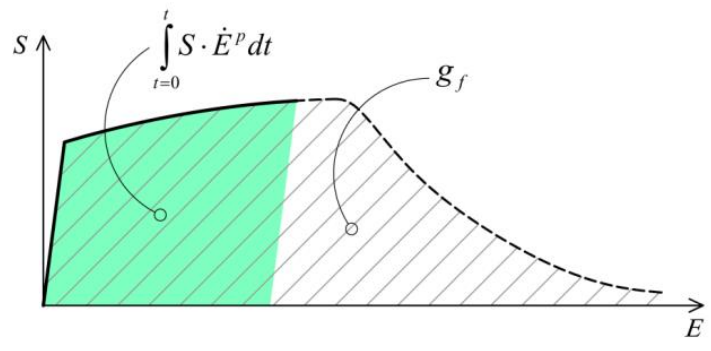

Figure 4. Representation of the volumetric fracture energy of a metallic material

Using the definition of the hardening internal variable defined in equation (21), it is possible to define the expression of the hardening function as:

$$
K=S^{e q}\left(\kappa^{p}\right)
$$

It can be easily proven that the hardening function and internal variable defined in equations (21) and

(22) fulfill the rate equations (11). The $h_{k}$ and $h_{\mathrm{k}}$ functions defined in expression (11) become:

$$
\begin{aligned}
& h_{k}=\frac{\partial S^{e q}}{\partial \kappa^{p}} \\
& h_{\mathrm{k}}=\frac{S}{g_{t}}
\end{aligned}
$$

\subsection{Expressions of the hardening function}

In this section the exact numerical expressions used to define the new hardening law presented in this work are provided. This law is characterized with two different functions, each one defining the evolution of the equivalent stress in each region in which the equivalent stress performance is divided (see Figure 3). 


\section{Region 1: Curve fitting with polynomial}

The first region is characterized with a polynomial defined by curved fitting from a given experimental data. Among the different available methods that can be used to define this polynomial, here is proposed to use the least squares method due to its simplicity, computational cost, and good performance provided. The resulting relation between the stress and plastic strain in this region is:

$$
S^{e q}\left(E^{p}\right)=a_{0}+\sum_{i=1}^{N} a_{i} \cdot\left(E^{p}\right)^{i}
$$

with $N$ the order of the polynomial.

The volume fracture energy that is dissipated in this region can be obtained calculating the area below the $S^{e q}-E^{p}$ graph. This calculation provides the following value:

$$
g_{t 1}=\sum_{i=1}^{N+1} \frac{a_{i}}{i}\left(\left(E_{2}^{p}\right)^{i}-\left(E_{1}^{p}\right)^{i}\right)
$$

being $E_{1}^{p}$ and $E_{2}^{p}$ the initial and final plastic strain values, respectively, that limit the polynomial function region.

Although the equivalent plastic stress should depend on the plastic internal variable $\kappa^{p}$, in a cyclic simulation with isotropic hardening, this approach will produce hysteresis loops with increasing stress amplitude (for a fixed strain amplitude). For this reason, current formulation calculates the equivalent plastic stress using the value of the equivalent plastic strain, which is obtained as:

$$
E^{p}=\frac{S: E^{p}}{f(S)}
$$

with $f(S)$ defined by the yield surface used to simulate the material, as it is shown in equation (8).

Finally, the derivative of the hardening function can be calculated with the following expression:

$$
\frac{d S^{e q}}{d \kappa^{p}}=\frac{d S^{e q}}{d E^{p}} \cdot \frac{d E^{p}}{d \kappa^{p}}=g_{t} \cdot \frac{\sum_{i=1}^{N} i \cdot a_{i+1}\left(E^{p}\right)^{i-1}}{\sum_{i=1}^{N+1} a_{i}\left(E^{p}\right)^{i-1}}
$$

Expression (27) is valid for values of $\kappa^{p}$ that are comprehended between $\kappa_{1}^{p}=0$ and $\kappa_{2}^{p}=g_{t 1} / g_{t}$. The value of the upper limit of the internal variable shows that it is necessary to define a value for the volumetric fracture energy of the material larger than $g_{t 1}$. If the value defined is lower, the material will not be able to reach its ultimate stress as this will imply having a fracture internal variable larger than 1.0 . 


\section{Region 2: Exponential softening}

When the plastic internal variable reaches the volumetric plastic energy available for the first region, $\kappa^{p}=\kappa_{2}^{p}$, isotropic hardening follows region two. The function that defines this new region is defined with the following parameters:

1. The initial equivalent stress value is defined by the equivalent stress reached in the first region $\left(S_{2}^{e q}\right)$. This value can be the one defined in the material characterization or can be a lower value if there has been some plastic energy dissipation in a cyclic process. In this last case, the stress value has to be obtained from the previous region.

2. The initial slope of the function is zero.

3. The volumetric fracture energy dissipated in this region is the remaining energy in the material:

$$
g_{t 2}=g_{t}-g_{t 1}
$$

With these considerations in mind, the resulting equation that relates the equivalent stress with the plastic strain is:

$$
S^{e q}\left(E^{p}\right)=S_{2}^{e q} \cdot\left[2 \cdot e^{-b \cdot\left(E^{p}-E_{2}^{p}\right)}-e^{-2 b \cdot\left(E^{p}-E_{2}^{p}\right)}\right]
$$

where $b=\frac{3 \cdot S_{2}^{e q}}{2 \cdot g_{t 2}}$

The expression of the equivalent stress as a function of the hardening variable is obtained combining equations (28) and (21), resulting:

$$
S^{e q}\left(\kappa^{p}\right)=S_{2}^{e q} \cdot \chi \cdot(2-\chi)
$$

being, $\chi=\sqrt{\frac{\left(\kappa^{p}-\kappa_{2}^{p}\right) \cdot 2 b \cdot g_{t}}{S_{2}^{e q}}+1}$

And the derivative of the hardening function is:

$$
\frac{d S^{e q}}{d \kappa^{p}}=2 b \cdot g_{t} \cdot\left(\frac{1}{\chi}-1\right)
$$


In the following, the results obtained from several simulations conducted to validate the formulation previously presented are included. This validation has been done comparing the numerical results with some of the experimental results obtained in the framework of the Ultra Low Cycle Fatigue Project ([42]).

\subsection{Description of the experimental tests}

Monotonic and cyclic tests were performed in a close-loop servo-hydraulic machine, INSTRON 8801, rated to $100 \mathrm{kN}$. The tests were performed at room-temperature in air. The fatigue tests were conducted under constant strain amplitudes and with a frequency adjusted to result an average strain rate of $0.008 \mathrm{~s}^{-1}$. The longitudinal strain was measured using a clip gauge with limit displacements of $\pm 2.5 \mathrm{~mm}$ with a gauge length of $12.5 \mathrm{~mm}$ (INSTRON 2620-602). This clip gauge was also used in two monotonic tensile tests allowing the registration of the longitudinal strains until approximately $17 \%$.

All tested specimens were machined according the longitudinal direction of 6" pipes made of X52 steel. The dimensions of the specimens are in accordance with the ASTM E606 standard, as illustrated in Figure 5. The shown specimen corresponds to the smooth plane (SP) series. The side faces of the specimens were milled and finished in order to remove the circumferential pipe curvature as well as surface imperfections. In order to achieve larger strain values in the specimen some of them where machined in order to reduce their section in their middle, using an oval hole in its center $(\mathrm{OH})$, as shown in Figure 6 . The experimental results obtained for this specimen have been also used to validate the model performance.

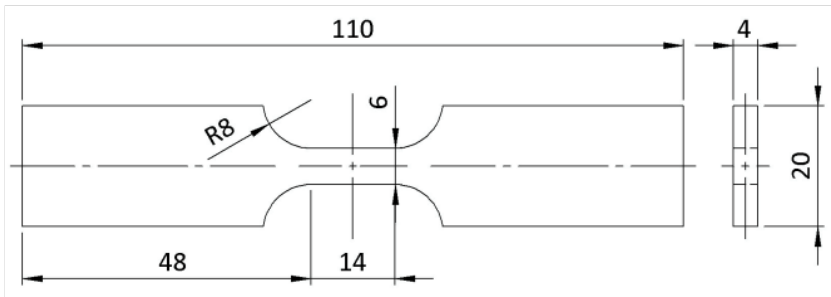

Figure 5. Geometry and dimensions, in millimeters, of the SP specimen

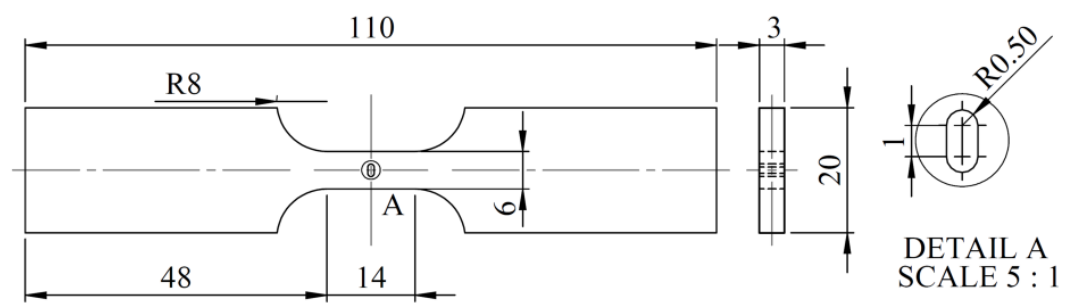

Figure 6. Geometry and dimensions, in millimeters, of the $\mathrm{OH}$ specimen 


\subsection{Description of the numerical models}

Two different numerical models have been defined, one for each experimental specimen. Figure 7 shows the meshes of both models. The SP model is made with 1608 quadratic hexahedral elements and 8839 nodes. It has three elements along its thickness and 563 elements on the face shown in Figure 7 (YZ plane). The $\mathrm{OH}$ model has 3080 quadratic hexahedral elements and 15460 nodes. It has five elements along the thickness and 616 elements in the $\mathrm{YZ}$ face. This second model requires nearly doubling the number of elements because the element size has to be significantly smaller around the hole for its correct simulation. The SP model has marked two red dots that were used to calculate the applied strain.
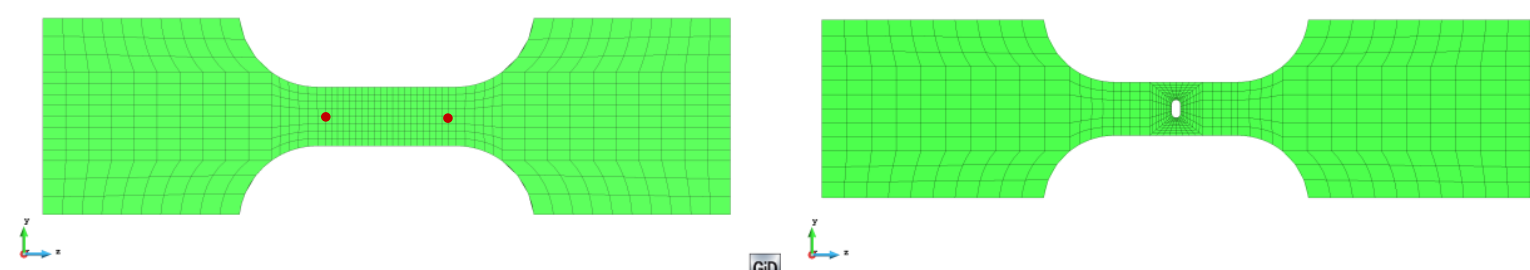

Figure 7. Meshes defined for the SP and $\mathrm{OH}$ numerical models.

As boundary conditions, the left border of the model has the displacement fixed to zero in all its directions, while the right border is moved with an imposed displacement in the sample longitudinal direction. The reaction force is obtained as a result of the numerical analysis.

All samples analyzed are defined with the same plastic material, defined with an associated plasticity and Von-Mises as yield law. The material properties are obtained with the calibration process that is described in the following section. All the material properties required by the model are displayed in Table 1. A parameter of this table that may seem surprising is the value of the yield stress, which is lower than the one expected for an X52 steel. This value is required because, if the yield stress is defined with a higher value, the kinematic hardening parameters lead to stress values too high in the hysteresis cycles. As the purpose of the model is the simulation of ULCF, which requires large plastic strain values, the requirement of defining a lower yield stress is not considered a major drawback. This yield stress does not represent the real material property but a numerical model parameter optimized to improve the description of the cyclic elasto-plastic behavior of the material.

The hardening region is defined with a polynomial of order five which is computed with the least squares method using available the experimental data. The constants of this polynomial, following notation shown in equation (24), are shown in Table 2. In order to define the polynomial constants, it has been necessary to take into account that the effects of the kinematic and the isotropic hardening laws are coupled. This implies that the definition of the first region of isotropic hardening cannot be obtained from the experimental curve straightforward, as this curve does not take into account the displacement of the yield surface due to the kinematic hardening law. 
Table 1. Numerical model parameters proposed for the X52 steel

\begin{tabular}{|lll|}
\hline Young Modulus & 180 & $\mathrm{GPa}$ \\
Poisson Ratio & 0.30 & \\
Yield Stress $\left(\sigma_{Y}^{e q}\right)$ & 240 & $\mathrm{MPa}$ \\
Plastic Strain threshold $\left(E_{2}^{p}\right)$ & 13 & $\%$ \\
c1 kinematic hardening param. & 60 & $\mathrm{GPa}$ \\
d1 kinematic hardening param. & 280 & \\
Fracture Energy $\left(G_{f}\right)$ & 1.9 & $\mathrm{MN} \cdot \mathrm{m} / \mathrm{m}^{2}$ \\
\hline
\end{tabular}

Table 2. Polynomial constants used to describe the hardening region of X52 material (Pa)

\begin{tabular}{|ll|}
\hline$a_{0}$ & $2.4000 \mathrm{E}+08$ \\
$a_{1}$ & $8.0084 \mathrm{E}+07$ \\
$a_{2}$ & $-1.1143 \mathrm{E}+08$ \\
$a_{3}$ & $8.7400 \mathrm{E}+07$ \\
$a_{4}$ & $-3.0507 \mathrm{E}+07$ \\
$a_{5}$ & $3.9073 \mathrm{E}+06$ \\
\hline
\end{tabular}

\subsection{Calibration of the numerical model}

The material data previously exposed has been obtained by model calibration. This is, adjusting the different parameters required by the model to obtain a good fitting with one of the experimental results available. For the current case, the experimental results considered are those of the SP specimen loaded with an imposed strain range of $2.75 \%$.

The results used to conduct the material calibration are the equivalent stress-equivalent strain graph obtained from the experimental test. The stress is computed as the total force applied to the specimen divided by the area of the cross section. The strains are computed dividing the measured displacement of the clipped gauge by the length of the gauge. In the numerical model these two parameters were calculated following the same procedure, using the red dots depicted in Figure 7.

The initial material parameters used in the calibration process are the ones commonly found in literature and the polynomial constants correspond to the ones obtained by curve fitting of the experimental results, ignoring the effect of kinematic hardening. Based on the strain-stress graph obtained with these first values, the different parameters shown in Table 1 and Table 2 have been modified (trial and error iterative procedure) until obtaining a numerical result that fits the experimental results.

Figure 8 shows the stress-strain graph provided by the two experimental samples tested and by the numerical model, which uses the material parameters obtained from the calibration process and 
described in Table 1 and in Table 2. As it can be seen, the agreement in the cyclic behavior of the numerical and experimental samples is rather good. This agreement is not achieved in the first monotonic loading excursion, as the model developed is not prepared to reproduce the initial plateau defined by the material. However, this disagreement is not considered relevant, as the model has been developed thinking on the cyclic behavior and the evolution of plastic response for larger plastic strain values, found beyond this initial plateau.

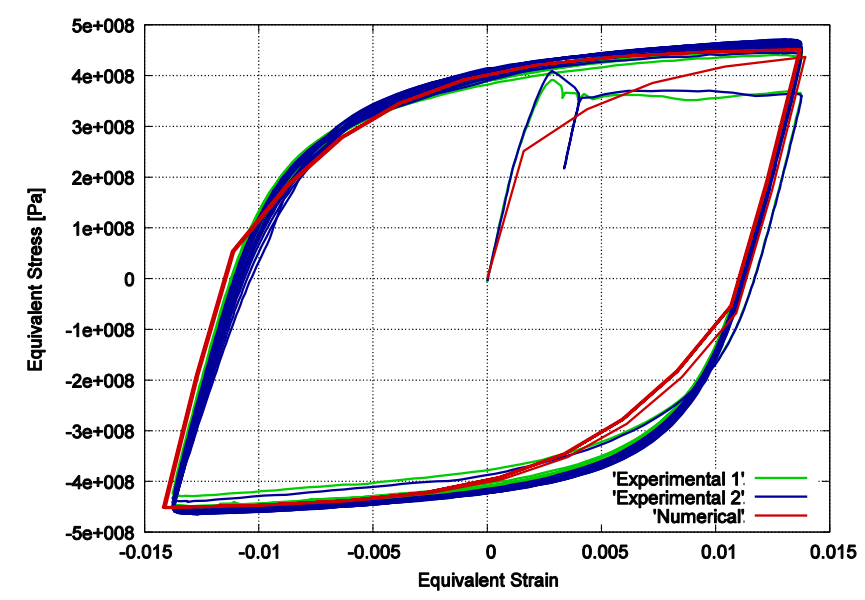

Figure 8. Stress-strain graphs for the SP sample with an applied deformation range of $2.75 \%$

The fitting of the stress-strain graph allows defining all material parameters except the fracture energy of the material and the equivalent plastic strain value at which softening is expected to start $\left(E_{2}^{p}\right)$. These two parameters have been defined to match the number of cycles that can be applied to the specimen before it fails (inverse calibration). In the experimental campaign, the first specimen failed after 150 cycles, and the second specimen failed after 103 cycles. To match these values, the numerical model has been defined with a maximum hardening strain $\left(E_{2}^{p}\right)$ of a $13 \%$. Afterwards, the material fracture energy parameter, $G_{f}$, is fitted until finding a value that predicts the failure of the numerical specimen after 128 cycles. These values are shown in Table 1 and used in the numerical simulations. Material failure is found when some gauss points reach a plastic damage value close to one. At this stage the numerical analysis cannot reach convergence and the specimen can be considered to be completely broken. In current simulation, the convergence is lost for a $\kappa^{p}$ value close to 0.9 , as it is shown in Figure 9, where the value of plastic damage is represented in the last tensile and compressive stages. 


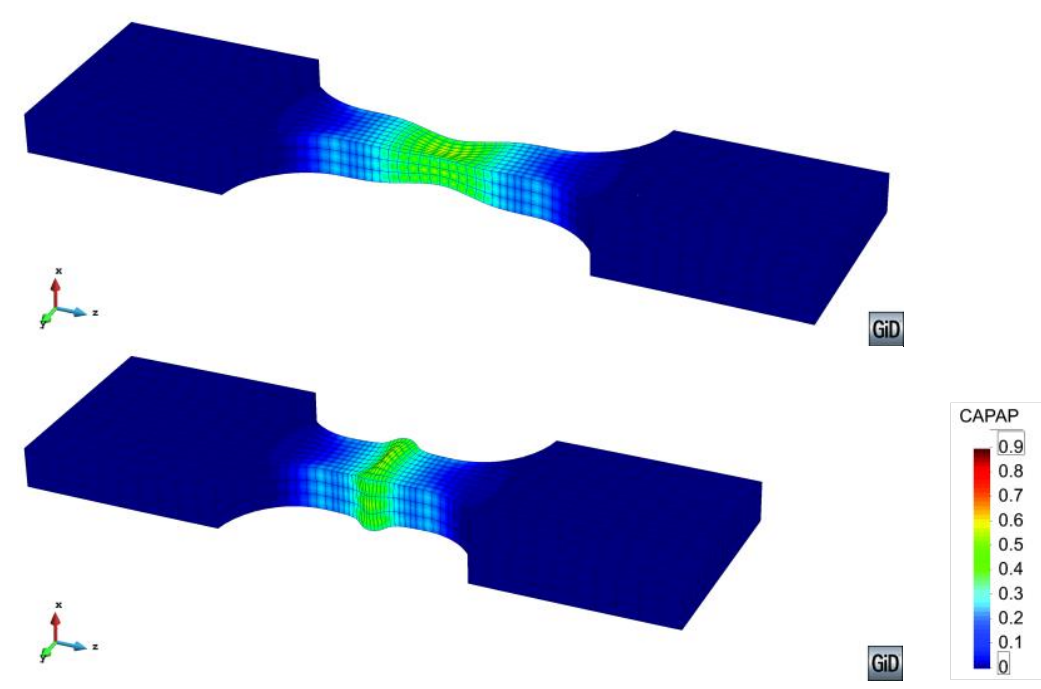

Figure 9. Values of $\kappa^{p}$ for the SP sample with an applied deformation range of $2.75 \%$, when applying the maximum tensile and compression strain before ULCF failure. Deformation is magnified by 20.

The mechanical performance of the numerical specimen along the simulation is shown in Figure 10, where the stress-strain loops for different cycles are represented. This figure shows that the effect of material softening in the specimen consists in a reduction of the equivalent stress obtained as the number of cycles applied to the specimen increase. The figure also shows that softening has already started in cycle 60, although the difference between the first loop and loop 60 is very small. In further cycles the effect of softening becomes more visible, until material fails in cycle 128, where the reduction of equivalent stress is close to a $20 \%$. Figure 9 also shows that the strain amplitude increases with the number of cycles applied to the specimen. This increase is obtained because equivalent strain is calculated as the relative displacement of two given nodes (red dots in Figure 7), divided by the original length between both nodes, and does not take into account that it is modified due to the plastic deformations existing in the whole specimen.

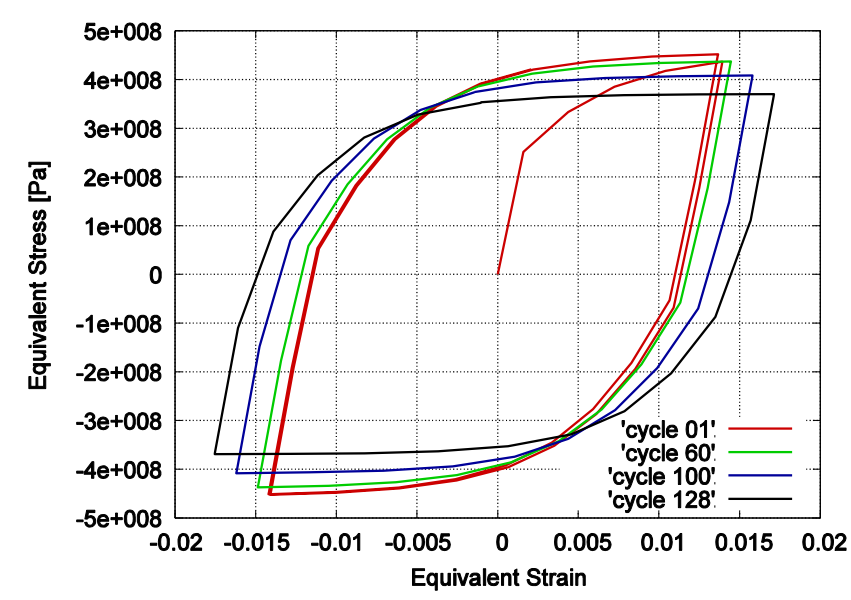

Figure 10. Evolution of the stress-strain relation for different cycles of the SP numerical analysis. 
The results obtained with the calibration model prove that the constitutive law proposed can be adjusted to any given stress-strain law by adjusting properly the material parameters. The calibration model has also been used to show the numerical and mechanical performance of the formulation developed. The prediction capacity of the model will be shown in further simulations, in which the material parameters are fixed and are used to analyze different specimens and load cases.

\subsection{Validation of the developed theory}

After having calibrated the material parameters, the rest of experimental tests available have been simulated in order to compare the ULCF failure prediction made by the numerical model with the results obtained from the experimental campaign. The constitutive model proposed in this work will succeed if it is capable of representing accurately the equivalent stress-strain graphs for the different deformation ranges tested experimentally and, even more important, if it is capable of predicting the number of cycles that can be applied to the specimen before its failure.

Figure 11 shows the stress-strain graph corresponding to the experimental and numerical SP sample with an applied equivalent strain of $4 \%$. In order to achieve this strain value, and to minimize the buckling of the specimen, the experimental test is conducted only on the positive strain region and with an antibuckling device. As can be seen, the experimental and the numerical results are in good agreement of the cyclic region of the curve. So, with this example it is proved that the model is capable of reproducing large strain values and non-symmetric cyclic patterns.

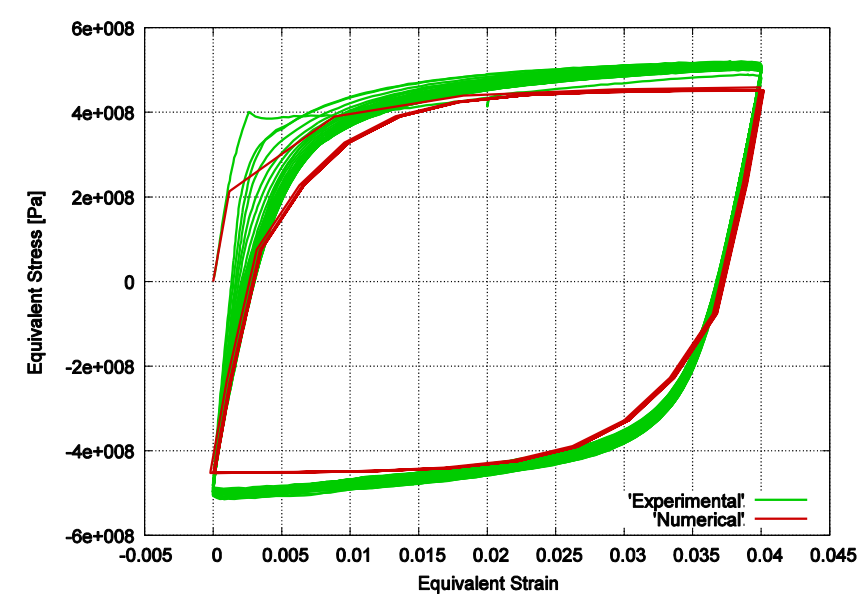

Figure 11. Stress-strain graphs for the SP sample with an applied deformation range of $4.00 \%$

Finally, Figure 12 compares the stress-strain experimental-numerical results obtained for one of the $\mathrm{OH}$ samples. The stress value for the $\mathrm{OH}$ samples is computed with the same procedure used for the SP samples, dividing the total force applied by the area of the cross section where the notch is found and the strain value is obtained dividing the clip gauge described in section 4.2. In this case, the numerical result prediction falls a bit shorter in terms of equivalent stress when compared to the experimental test. However, a closer look to the experimental curve shows that in the first loading 
branch the experimental and numerical tests match perfectly, and it is in further cycles that the experimental test provides larger equivalent stress values. This difference between hysteresis loops are explained by an initial isotropic hardening of the material, for high cyclic plastic strain ranges experienced by the material around the notch (oval hole), which cannot be captured by the proposed model since the isotropic hardening was calibrated for lower plastic strain ranges, where this hardening effects are not so pronounced. Since the material shows an isotropic hardening that is dependent on the plastic strain range experienced by the material, the calibration of the material should take into account the expected range of plastic strain ranges to be simulated. Despite this difference, results are considered similar enough to validate the material parameters defined in the numerical model calibration.

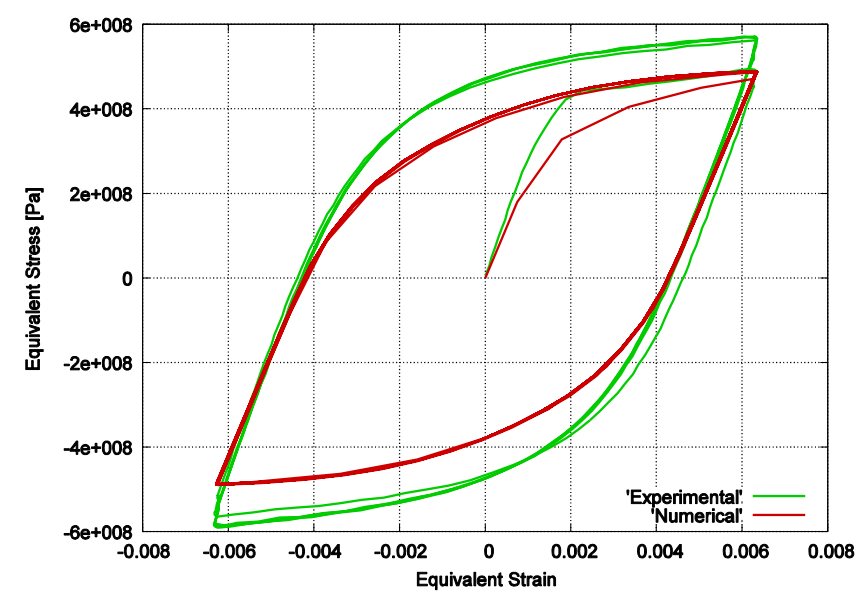

Figure 12. Stress-strain graphs for the $\mathrm{OH}$ sample with an applied deformation range of $1.25 \%$

Once having proved that the developed formulation is capable of reproducing the mechanical response obtained with the experimental samples, the following step is to verify if the formulation is capable of predicting the failure of the specimens due to Ultra Low Cycle Fatigue. This validation is performed counting the number of cycles that can be applied to the numerical model before its failure. The numerical model is considered to fail when the convergence of the analysis is lost. This occurs when some gauss points reach a $\kappa^{p}$ value close to 1.0 . The number of cycles applied to the numerical model are compared with the cycles obtained in the experimental campaign.

Figure 13 shows the results obtained for the SP samples. Results with reversion strain factor of -1 and of 0 are plotted together because the reversion factor does not play a significant role in the material response to ULCF. This figure shows that the number of cycles to failure predicted by the numerical simulation are in very good agreement with the number of cycles obtained in the experimental campaign. The only value that is not contained between the experimental results is the one corresponding to an applied strain range of $3.5 \%$. However, the value provided by the numerical simulation looks more coherent than the one obtained with the experimental test, as the number of cycles obtained in the experimental test is larger than the one obtained for an applied strain range of $2.75 \%$. 


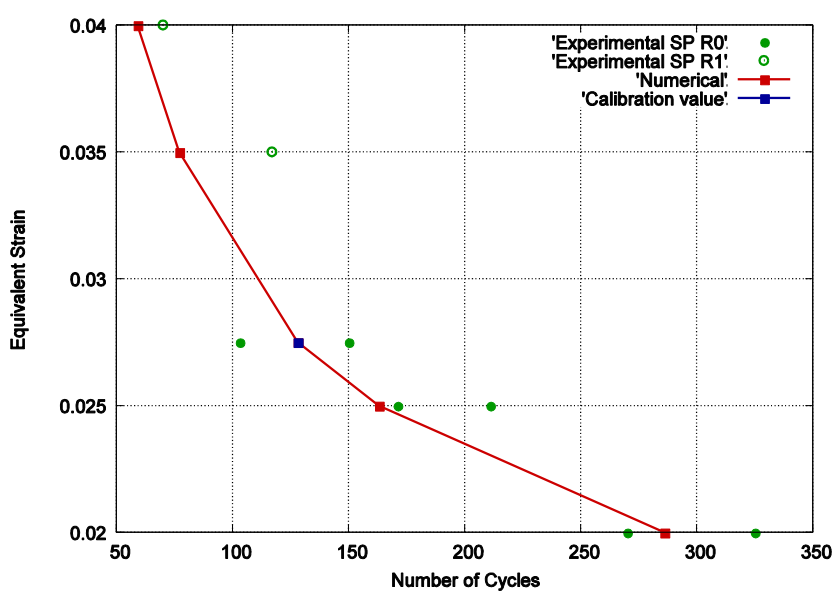

Figure 13. ULCF failure prediction for SP specimens

The results obtained for the $\mathrm{OH}$ samples are shown in Figure 14. For these samples the experimental test was conducted in just one specimen for each strain value, therefore there is no possibility of knowing the scatter expected in the experimental tests. However, the number of cycles predicted by the formulation is, for all strains, in the same order of magnitude than the experimental results obtained. Therefore, it can be concluded that the formulation is, again, capable of predicting accurately the ULCF failure of the $\mathrm{OH}$ specimens.

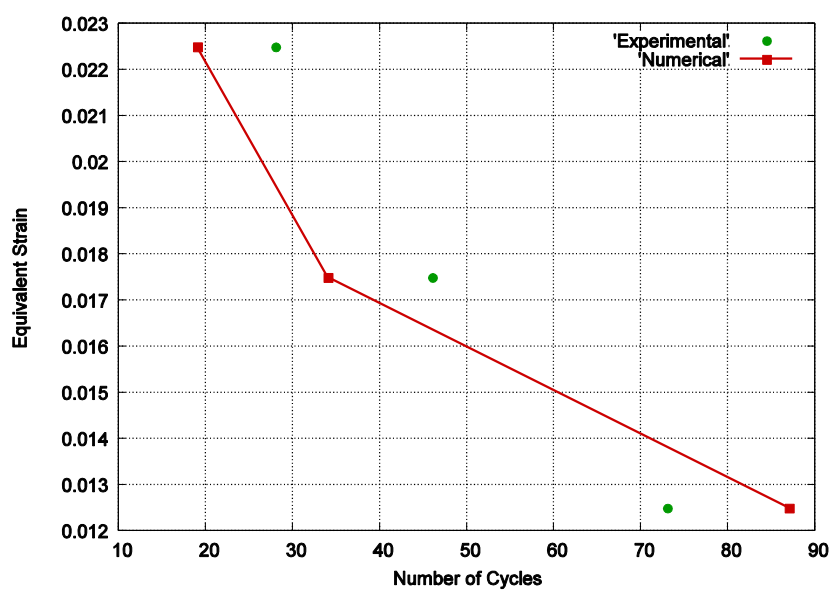

Figure 14. ULCF failure prediction for $\mathrm{OH}$ specimens

It is important to remark that the material properties used for all numerical simulations are exactly the same. Therefore, the variation in the prediction of the number of cycles that can be applied to any of the specimens considered is a result of the energy dissipated in each case. The agreement obtained in all cases, independently of the reversion factor or the stress concentrations due to the existing oval hole (OH specimens) allows considering the approach used to characterize ULCF failure an excellent option. Moreover the formulation allows conducting simulations in which the cycles can be nonregular, with varying amplitude and frequency, in which there can be sustained monotonic loads between cycles or, in general, in which the load applied is not a regular one. This capability is not offered by any other formulation available. 


\subsection{Advantages of the approach proposed}

Previous results have shown that the proposed constitutive model is capable of predicting material failure after applying several cycles to the material. However, this prediction capability does not present a major advantage compared to other approaches such as the Coffin-Manson rule, or any other analytical expression capable of defining the maximum number of cycles that can be applied for a given plastic strain.

The main advantage of the proposed approach is that the prediction of ULCF failure does not depend on the applied plastic strain, but on the energy dissipated during the cyclic process. Therefore, it is possible to vary the plastic strain in the cycles applied to the structure and the constitutive model will still be capable of predicting the material failure.

This is proved in the following example, where an irregular load, in frequency and amplitude, is applied to the SP specimen defined in Section 4.2 and used to validate the formulation. The load defined is depicted in Figure 15a. This load is applied as a fixed displacement following the same procedure used for the SP specimen. The stress strain graph obtained from the numerical model is plotted in Figure 15b. As it can be seen, the applied load produces several loops, each one with a different plastic strain.

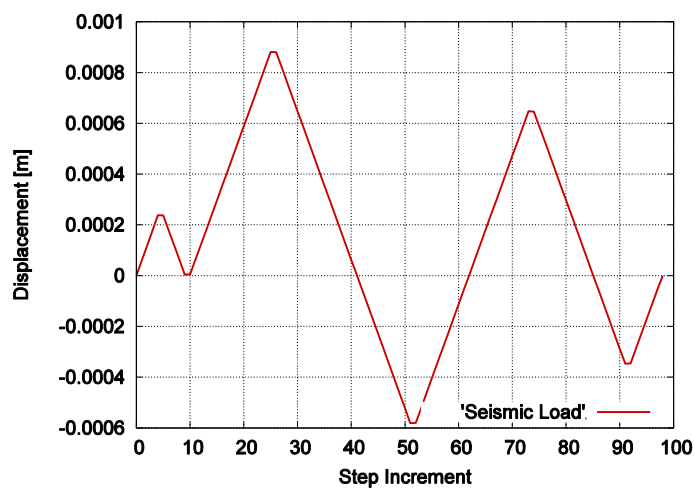

(a)

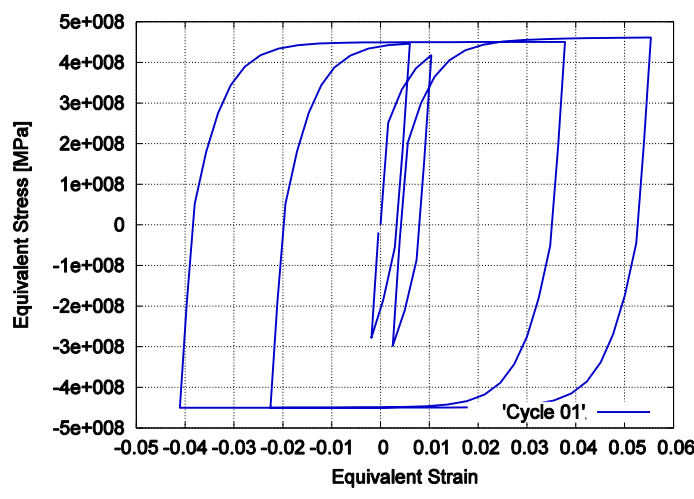

(b)

Figure 15. Seismic-type load applied (a) and material stress-strain response (b)

The model is capable of capturing the energy dissipated in each one of these loops and, therefore, to evaluate the energy available in the material after having applied the load, which is equivalent to the residual strength of the material. It is also possible to repeat several times the irregular load block, shown in Figure 15a, to study the number of repetitions that are required to reach material failure. Figure $16 \mathrm{~b}$ shows the stress-strain response of the material after 10 blocks similar to the Figure 15a (Figure 16a). At this point, there are some points in the model that have lost most of their fracture strength and specimen failure occurs. As occurred with the SP model, this simulation also shows some lateral displacement on the equivalent strains due to the plastic strains suffered by the whole specimen. 


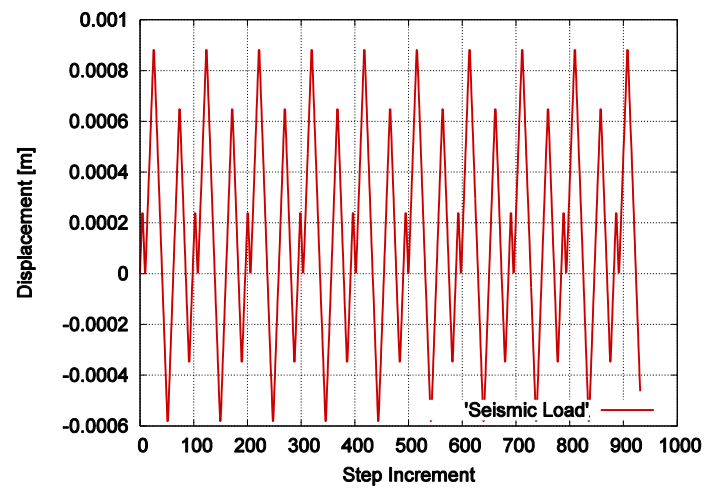

(a)

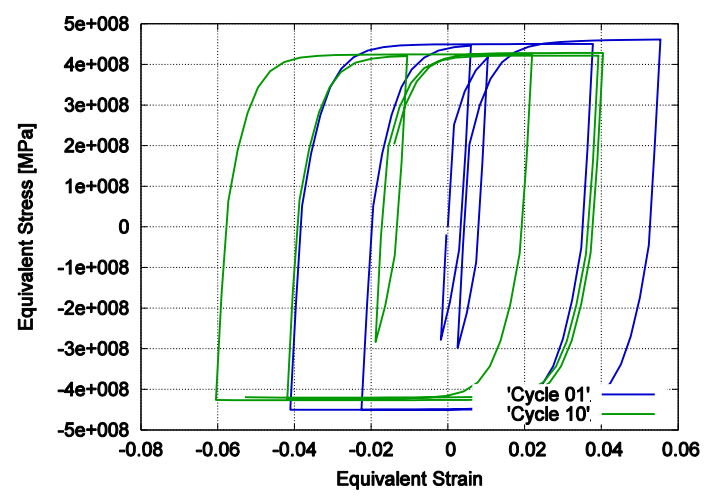

(b)

Figure 16. Response of the model after ten seismic-type cycles: a) displacement-time history; b) stressstrain history.

\section{CONCLUSIONS}

This work has presented a new plastic-damage formulation specially developed to simulate the mechanical response of steel, and its failure due to Ultra Low Cycle Fatigue (ULCF). The formulation is based on the Barcelona plastic model initially proposed by Lubliner et al. [1], which has been improved by adding a non-linear kinematic hardening law coupled with a new isotropic hardening law. The isotropic hardening law is divided in two regions. In the first one the steel exhibits a hardening behavior, this region is defined by several points that have to be obtained from experimental tests. And, in the second region, the steel has a softening behavior, which is defined with an exponential law. The evolution of the material in these two regions is driven by the fracture energy that can be dissipated by the material.

This approach allows predicting material failure by the constitutive model on its own, without the need of additional parameters or additional laws specially chosen for the failure criteria that wants to be simulated. Therefore, with the proposed formulation it is possible to simulate accurately the mechanical response of steel under different loading scenarios, such regular or ramdom cyclic loading. This last case is equivalent to the load that will be obtained in a seismic case, where ULCF may be one of the main causes of structural failure. Several numerical analyses have been performed in order to show the behavior of the formulation under the different loading scenarios mentioned.

The capacity of the formulation to simulate accurately the ULCF phenomenon has been proved by reproducing different experimental tests made on X52 steel samples. The experimental campaign consisted in loading several smooth specimens with different strain amplitudes. Tests were also performed to notched specimens in order to increase the plastic strain and reduce the number of cycles that could be applied before failure. One of the experimental tests has been used to calibrate the material parameters of the model; afterwards all other specimen behaviors have been reproduced 
numerically. All the numerical results obtained with the proposed constitutive model have provided an excellent agreement with the experimental tests, proving the validity of the proposed formulation to simulate the plastic response of steel and its failure due to Ultra Low Cycle Fatigue.

\section{Acknowledgements}

This work has been supported by the Research Fund for Coal and Steel through the ULCF project (RFSR-CT-2011-00029), by the European Research Council under the Advanced Grant: ERC-2012-AdG 320815 COMP-DES-MAT "Advanced tools for computational design of engineering materials", and by the research collaboration agreement established between Abengoa Research and CIMNE. All this support is gratefully acknowledged.

\section{REFERENCES}

[1] J. Lubliner, J. Oliver, S. Oller and E. Oñate. A plastic-damage model for concrete. International Journal of Solids and Structures, 25(3): 299-326 (1989)

[2] S. Oller, O. Salomón, E. Oñate. A continuum mechanics model for mechanical fatigue analysis. Computational Materials Science, 32(2): 175-195 (2005)

[3] S. Oller, A. Suero. Tratamiento del Fenómeno de Fatiga Isotérmica Mediante la Mecánica de Medios Continuos. Revista Internacional de Métodos Numéricos para el Cálculo y diseño en Ingeniería, 15(1): 3-29. (1999)

[4] L.G. Barbu, S. Oller, X. Martínez, A.H. Barbat. Stepwise advancing strategy for the simulation of fatigue problems. Complas XII, Barcelona, 3-5 September (2013)

[5] ASTM Standard E1823-13. Standard Terminology Relating to Fatigue and Fracture Testing. ASTM International, West Conshohocken, PA (2013)

[6] A.M Kanvinde, G.G. Deierlein. Micromechanical simulation of earthquake-induced fracture in steel structures. Technical Rep. 145, John A. Blume Earthquake Engineering Center, Stanford University, Calif. (2004)

[7] L Xue, A unified expression for low cycle fatigue and extremely low cycle fatigue and its implication for monotonic loading, Int. J. Fatigue, 30, 1691-1698 (2008)

[8] F.C. Campbell, Elements of Metallurgy and Engineering Alloys, ASM International, Ohio, USA (2008)

[9] J.T.P. Yao and W.H. Munse. Low-cycle fatigue on metals - Literature review. Technical Report SSC137. Ship Structure Committee (1963) 
[10] H.D. Solomon, G.R. Halford, L.R. Kaisand, B.N. Leis. Low Cycle Fatigue: Directions for the Future. ASTM STP942 technical papers (1988)

[11] M. Kuroda. Extremely low cycle fatigue life prediction based on a new cumulative fatigue damage model. International Journal of Fatigue, 24(6): 699-703 (2002)

[12] K. Tateishi, T. Hanji, K. Minami. A prediction model for extremely low cycle fatigue strength of structural steel International. Journal of Fatigue, 29(5): 887-896 (2007)

[13] Y. Jiang, W. Ott, C. Baum, M. Vormwald, H. Nowack, Fatigue life predictions by integrating EVICD fatigue damage model and an advanced cyclic plasticity theory. International Journal of Plasticity, 25(5): 780-801 (2009)

[14] H. Nowack, D. Hanschmann, W. Ott, K.H. Trautmann, E. Maldfeld, Crack initiation life behaviour under biaxial loading conditions: experimental behaviour and prediction. Multiaxial Fatigue and Deformation Testing Techniques, 12: 159-183 (1996)

[15] W. Ott, O. Baumgart, K.H. Trautmann, H. Nowack, A new crack initiation life prediction method for arbitrary multiaxial loading considering mean stress effect. In: Lüdjering, G., Nowack, H. (Eds.), Proceedings of the 6th International Fatigue Conference (FATIGUE '96), Berlin, Germany, pp. 1007-1012 (1996)

[16] W. Ott, C.C. Chu, K.H. Trautmann, H. Nowack. Prediction capability of a new damage event independent (continuous) multiaxial fatigue prediction method (EVICD-N). In: Ellyn, F., Provan, W. (Eds.), Proc. 8th Int. Conf. Mech. Behaviour Mat. (ICM 8), Progress in Mechanical Behaviour of Materials, Victoria, Canada, pp. 1204-1209 (2000)

[17] A.M. Kanvinde, G.G. Deierlein, Void growth model and stress modified critical strain model to predict ductile fracture in structural steels. Journal of Structural Engineering, 132(2): 1907-1918 (2006)

[18] A.M. Kanvinde, G.G. Deierlein, Cyclic void growth model to assess ductile fracture initiation in structural steels due to ultra low cycle fatigue. Journal of Engineering Mechanics, 136(6): 701-712 (2007)

[19] K. Saanouni, A. Abdul-Latif, Micromechanical modeling of low cycle fatigue under complex loading - Part I. Theoretical formulation. International Journal of Plasticity, 12(9): 1111-1121 (1996)

[20] A. Abdul-Latif, K. Saanouni. Micromechanical modeling of low cycle fatigue under complex loadings - Part II. Application. International Journal of Plasticity, 12(9): 1123-1149 (1996)

[21] M. Naderi, S.H. Hoseini, M.M. Khonsari. Probabilistic simulation of fatigue damage and life scatter of metallic components. International Journal of Plasticity 43: 101-115 (2013)

[22] J. Lemaitre and J.-L. Chaboche. Mechanics of Solid Materials. Cambridge University Press. New York, USA, 1990 
[23] A. Warhadpande, B. Jalalahmadi, T. Slack, F. Sadeghi. A new finite element fatigue modeling approach for life scatter in tensile steel specimens. International Journal of Fatigue 32(4): 685-697 (2010)

[24] S. Oller, J. Oliver, J. Lubliner, E. Oñate. Un Modelo Constitutivo de Daño Plástico Para Materiales Friccionales: Parte I, Variables Fundamentales, Funciones de Fluencia y Potencial. Revista Internacional de Métodos Numéricos para el Cálculo y Diseño en Ingeniería, 4(4): 397-431 (1988).

[25] S. Oller, J. Oliver, J. Lubliner, E. Oñate. Un Modelo Constitutivo de Daño Plástico Para Materiales Friccionales: Parte II, Generalización para Procesos con Degradación de Rigidez, Ejemplos. Revista Internacional de Métodos Numéricos para el Cálculo y diseño en Ingeniería, 4(4): 433-461 (1988)

[26] J. Lee, G. Fenves. Plastic-Damage Model for Cyclic Loading of Concrete Structures. Journal of Engineering Mechanics, 124(8): 892-900 (1998)

[27] X. Martinez, S. Oller, L.G. Barbu, A.H. Barbat A.H. Analysis of Ultra Low Cycle Fatigue problems with the Barcelona plastic damage model. Complas XII, Barcelona, 3-5 September (2013)

[28] L.G. Barbu, S. Oller, X. Martinez, A.H. Barbat. Coupled plastic damage model for low and ultra-low cycle seismic fatigue. 11th World Congress on Computational Mechanics (WCCM XI) Barcelona 20-25 July. (2014)

[29] B. Luccioni, S. Oller and R. Danesi. Coupled plastic-damage model. Computer Methods in Applied Mechanics and Engineering, 129: 81-90 (1996)

[30] S. Oller. Fractura mecánica. Un enfoque global. Centre Internacional de Mètodes Numèrics a I'Enginyeria (CIMNE). Barcelona, Spain, 2001. ISBN: 84-89925-76-3

[31] J. Lubliner. On thermodyamics foundations of non-linear solid mechanics. International Journal non-linear Mechanics. 1972, 7: 237-254

[32] J. Lubliner. Plasticity Theory. Macmillan Publishing. New York, USA, 1990

[33] A. Green, and P. Naghdi. A general theory of an elastic-plastic continuum. Archive for Rational Mechanics and Analysis. 1964, 18(4): 251-281

[34] L. Malvern. Introduction to the Mechanics of Continuous Medium. Prentice Hall. New Jersey, USA, 1969. ISBN: 978-013487

[35] G. Maugin. The Thermomechanics of Plasticity and Fracture. Cambridge University Press. New York, USA, 1992

[36] B.K. Chun, J.T. Jinna and J.K. Lee. Modeling the Bauschinger effect for sheet metals. Part I: theory. International Journal of Plasticity. 2002, 18: 571-595

[37] M.E. Kassner, P. Geantil, L.E. Levine and B.C. Larson. Backstress, the Bauschinger Effect and Cyclic Deformation. Materials Science Forum. Vols. 604-605: 39-51. Trans Tech Publications. Switzerland, 2009 
[38] S. Oller. Nonlinear Dynamics of Structures. Springer, 2014. 208p. ISBN 978-3-319-05194-9

[39] X. Martinez, S. Oller, F. Rastellini and H.A. Barbat, A numerical procedure simulating RC structures reinforced with FRP using the serial/parallel mixing theory. Computers and Structures 2008, 86(15-16): 1604-1618

[40] Martinez, X., Oller, S. and Barbero, E., Mechanical response of composites. Chapter: Study of delamination in composites by using the serial/parallel mixing theory and a damage formulation. Springer, ECCOMAS series Edition, 2008

[41] X. Martínez, S. Oller, E. Barbero (2011). Caracterización de la delaminación en materiales compuestos mediante la teoría de mezclas serie/paralelo. Revista Internacional de Métodos Numéricos para Cálculo y Diseño en Ingeniería. 27(3):189-199, DOI:10.1016/j.rimni.2011.07.001. Elsevier. ISSN: 0213-1315.

[42] J.C.R. Pereira, A.M.P. de Jesus, J. Xavier, A.A. Fernandes, B. Martins, Comparison of the monotonic, low-cycle and ultra-low-cycle fatigue behaviors of the X52, X60 and X65 piping steel grades, Proceedings of the 2014 ASME Pressure Vessels \& Piping Conference, ASME 2014 PVP, July 20 24, 2014, Anaheim, California, USA 